\title{
Unscrambling Mixed Elements using High Angle Annular Dark Field Scanning Transmission Electron Microscopy
}

\author{
Karel H. W. van den Bos, ${ }_{1}^{1}$ Annick De Backer, ${ }^{1}$ Gerardo T. Martinez, ${ }^{1}$ Naomi Winckelmans, ${ }^{1}$ \\ Sara Bals, ${ }^{1}$ Peter D. Nellist, ${ }^{2}$ and Sandra Van Aert ${ }^{1, *}$ \\ ${ }^{1}$ EMAT, University of Antwerp, Groenenborgerlaan 171, 2020 Antwerp, Belgium \\ ${ }^{2}$ Department of Materials, University of Oxford, 16 Parks Road, Oxford OX1 3PH, United Kingdom
}

(Received 13 March 2016; revised manuscript received 16 May 2016; published 17 June 2016)

\begin{abstract}
The development of new nanocrystals with outstanding physicochemical properties requires a full threedimensional (3D) characterization at the atomic scale. For homogeneous nanocrystals, counting the number of atoms in each atomic column from high angle annular dark field scanning transmission electron microscopy images has been shown to be a successful technique to get access to this $3 \mathrm{D}$ information. However, technologically important nanostructures often consist of more than one chemical element. In order to extend atom counting to heterogeneous materials, a new atomic lensing model is presented. This model takes dynamical electron diffraction into account and opens up new possibilities for unraveling the 3D composition at the atomic scale. Here, the method is applied to determine the 3D structure of $\mathrm{Au} @ \mathrm{Ag}$ core-shell nanorods, but it is applicable to a wide range of heterogeneous complex nanostructures.
\end{abstract}

DOI: 10.1103/PhysRevLett.116.246101

The introduction of different atomic elements into nanocrystals can enhance properties such as stability, catalytic activity, and electrical response [1-3]. Since the exact threedimensional (3D) arrangement of atoms controls these properties, quantitative structure determination is essential for the development of new nanodevices. Counting the number of atoms in each atomic column from different viewing directions has proven to be a powerful technique to retrieve 3D information for homogeneous nanostructures $[4,5]$. Furthermore, the combination of atom counts from a single viewing direction with ab initio calculations [6] or molecular dynamics [7] enables the study of beam-sensitive materials and the study of particle dynamics at the atomic scale. High angle annular dark field (HAADF) scanning transmission electron microscopy (STEM) is used because of its ability to provide direct structural images with subangström resolution that are sensitive to both the thickness and the atomic number $Z$ [1,8-11]. In order to extend the atom-counting technique to heterogeneous materials, this Letter presents a new atomic lensing model facilitating both atom counting and 3D compositional determination in such materials. The power of this model is illustrated using experimental and simulated images of $\mathrm{Au} @ \mathrm{Ag}$ nanorods.

The so-called scattering cross section, corresponding to the total intensity of electrons scattered by a single atomic column, has been shown to be a successful performance measure for extracting structural information from HAADF STEM images [4,12-18]. This measure increases monotonically with thickness and, therefore, outperforms peak intensities which saturate beyond a certain thickness [19]. Furthermore, scattering cross sections are very robust for probe parameters like defocus, source coherence [13], and aberrations [20]. This quantity is measured either by integrating the image contribution of an atomic column $[13,21]$ or by estimating the volume under a Gaussian peak describing the shape of the image of an atomic column $[4,14,15]$. For a quantitative comparison with simulations, HAADF STEM images need to be recorded on a calibrated intensity scale [19,22-25]. In this manner, libraries of simulated scattering cross sections can be matched to experimental, calibrated scattering cross sections to characterize the projected atomic columns [7,13]. Van Aert et al. proposed an alternative statistics-based method to quantify the number of atoms with single-atom sensitivity $[4,14,15]$. It is then not required to have a calibrated detector or image simulations, because counting results are obtained by quantifying the intensities in a relative manner. It is expected that the combination of image simulations with the statisticsbased method gives the most reliable quantification [14].

So far, these approaches enabled one to count the number of atoms in homogeneous structures $[4,7,14,15]$ and to determine the chemical concentration in heterogeneous complex structures having a constant thickness $[12,16,17]$. Often, a linear dependence on concentration is assumed, although small changes in atom ordering in the column can modify the scattering cross sections [16,26,27]. An illustration of this effect is shown in Fig. 1(a), where the depth location of a single $\mathrm{Pt}$ atom in an eight-atom-thick column of Co modifies the scattering cross section. This depth dependency requires, however, a quantitative method capable of recognizing all possible 3D column configurations, already more than $2 \times 10^{6}$ for a 20-atom-thick binary alloy having all possible ratios between both elements. Image simulations play an important role for obtaining access to this information, but the amount of required simulations makes it an impossible task in terms of computing time. Therefore, a 
(a) $\underset{0}{\pi} 0.16$ simulated values

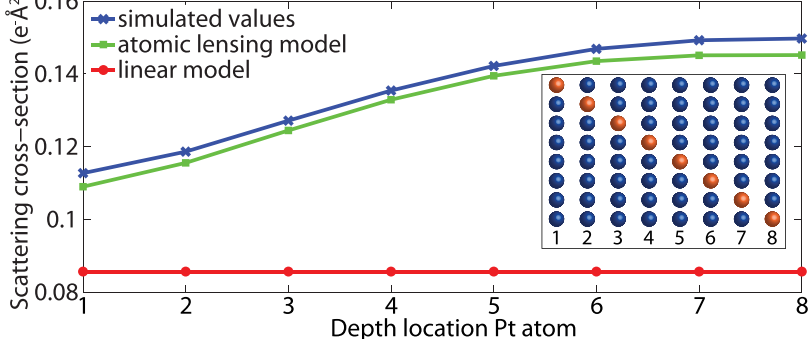

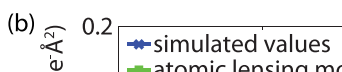

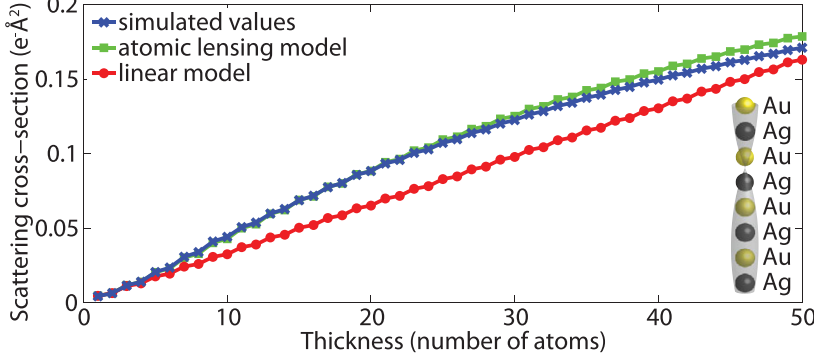

(c) $\underset{\text { 这 }}{0} 0.12 \#$ simulated values

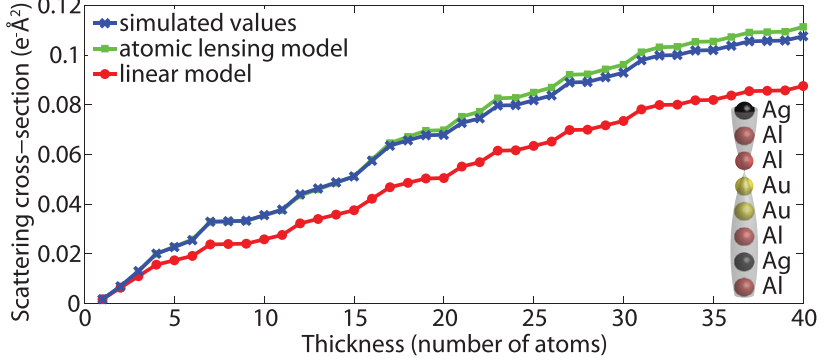

FIG. 1. The atomic lensing model for different column configurations compared to image simulations and a linear model neglecting channeling. (a) An eight-atom-thick column of $\mathrm{Co}$ atoms containing $1 \mathrm{Pt}$ atom at different depth locations. (b) A fcc $\mathrm{Au}-\mathrm{Ag}$ crystal in [110] zone-axis orientation, with $\mathrm{Au}$ and $\mathrm{Ag}$ atoms alternating. (c) A column composed of $\mathrm{Al}, \mathrm{Ag}$, and $\mathrm{Au}$ in a random ordering. The multislice approach with emission potential is used with parameters $U_{a}=300 \mathrm{kV}, C_{1}=-8.3 \mathrm{~nm}$, $C_{3}=0.035 \mathrm{~mm}, C_{5}=0 \mathrm{~mm}$, and $\alpha=22 \mathrm{mrad}$, and a detector collection range of $90-180 \mathrm{mrad}$ [28].

nonlinear model, capable of predicting scattering cross sections in terms of the 3D atomic arrangement, is developed by taking dynamical electron diffraction into account.

Dynamical electron diffraction in crystals can be understood from the so-called channeling theory, where each atom is considered as a thin, positive electrostatic lens focusing the incoming electrons along atom columns parallel to the beam direction [29]. In zone-axis orientation, the electron wave passes successive lenses focusing electrons at periodic distances. This period, called the extinction distance, is controlled by the atomic number, determining the strength of each lens, the atom spacing in the column, and the DebyeWaller factor [30,31]. In this model, the atomic column acts as a guide or channel trapping the electrons inside the column. This process is, however, complicated by the possibility that electrons can leave the column, for example, by thermal diffuse scattering [32]. For on-column probe positions, this effect is more pronounced than for scattering cross sections, as suggested by the leveling off of peak intensities with respect to thickness [19].

The proposed model, which will be used to unravel mixed elements, takes the lensing effect present in scattering cross sections into account by modeling it as a superposition of individual atoms focusing the incoming electrons; see also Fig. 2(a). This semiempirical model is justified by the channeling theory, where one can predict that the focusing of electrons of all atoms in a column causes a nonlinear increase of the scattering cross sections with thickness. Measuring this increase, by taking a first derivative, therefore quantifies the lensing effect of an atomic column as a whole, as illustrated in Fig. 2(b). Because this increase also depends on the atomic number of the last atom in the column [the red atom in Fig. 2(b)], the first derivative is normalized by the scattering cross section of a single freestanding atom. By using the superposition principle, the second derivative then gives the lensing effect of an individual atom; see also Fig. 2(c). The strength of such a physics-based model is that these values can be computed from image simulations of pure columns. Next, the superposition principle can be used to determine the lensing effect of any mixed column in order to predict its scattering cross section; see Fig. 2(a). A mathematical description can be found in the Supplemental Material [33].

The atomic lensing model is validated by comparisons with image simulations of different column configurations shown in Fig. 1. A linear model, neglecting channeling, is shown to highlight the importance of the atomic lensing model. In contrast to the linear model, the atomic lensing model preserves the dependency on the column configuration; see Fig. 1(a). Furthermore, the atomic lensing model is not restricted to a maximum number of atom types or a


FIG. 2. The atomic lensing model visualized. (a) The lensing effect in an atomic column modeled as the superposition of individual atoms focusing electrons on the column. (b) The normalization of the first derivative of the scattering cross section with thickness by the scattering cross section of a single freestanding atom gives the lensing effect of a column of atoms on an absolute scale. (c) Subtracting the lensing effect of different atomic columns gives the lensing effect of the individual atoms. 
specific column ordering, as shown in Figs. 1(b) and 1(c). Figure 1 demonstrates the excellent match with image simulations up to a thickness of about 25 atoms. Beyond this thickness, the underlying superposition model still holds approximately. Deviations are caused by the fact that the lensing effect of a specific atom in the column may be influenced by the presence of neighboring atoms. For example, the superposition model assumes that the second atom in a column does not modify the lensing effect of the first atom. The validity of this assumption will depend on the coherency of the signal, which is, among other factors, determined by the detection geometry [9,34]. The degree up to which atoms can affect the lensing effect of other atoms will become smaller if the signal becomes more incoherent. Therefore, the most accurate predictions for the atomic lensing model are expected in the HAADF regime.

The atomic lensing model is an excellent tool to demonstrate that scattering cross sections of different column configurations will be overlapping in the presence of noise. For example, the difference in scattering cross section is less than $1 \%$ for a five-atom-thick Co column containing $2 \mathrm{Pt}$ impurity atoms at the top and an eight-atom-thick Co column containing 1 Pt impurity atom at the center [depth location 4 in Fig. 1(a)]. This shows the need to include prior knowledge when one wants to extract the 3D structure from a limited number of projection images. The following examples demonstrate the type of a priori knowledge that can and should be exploited, if available, to retrieve the 3D atomic structure of heterogeneous systems.

In a first example, an experimental HAADF STEM image of a Au@Ag core-shell nanorod has been investigated. These nanorods have been extensively studied because of the possibility to tune their optical response, making them excellent building blocks for electro-optical devices and contrast agents in biomedical applications [35-40]. Images along two different viewing directions, shown in Fig. 3, have been recorded by using the X-Ant-EM, a probe-corrected FEI Titan ${ }^{3}$, operated at $300 \mathrm{kV}$. The HAADF regime has been selected by using a semiconvergence angle of $21 \mathrm{mrad}$ and detector collection range of 58-215 mrad [34]. In order to limit the number of variables that have to be predicted, the total thickness has been estimated. It is known that these nanorods yield a predominantly octagonal cross section [41]. However, electron tomography experiments demonstrate
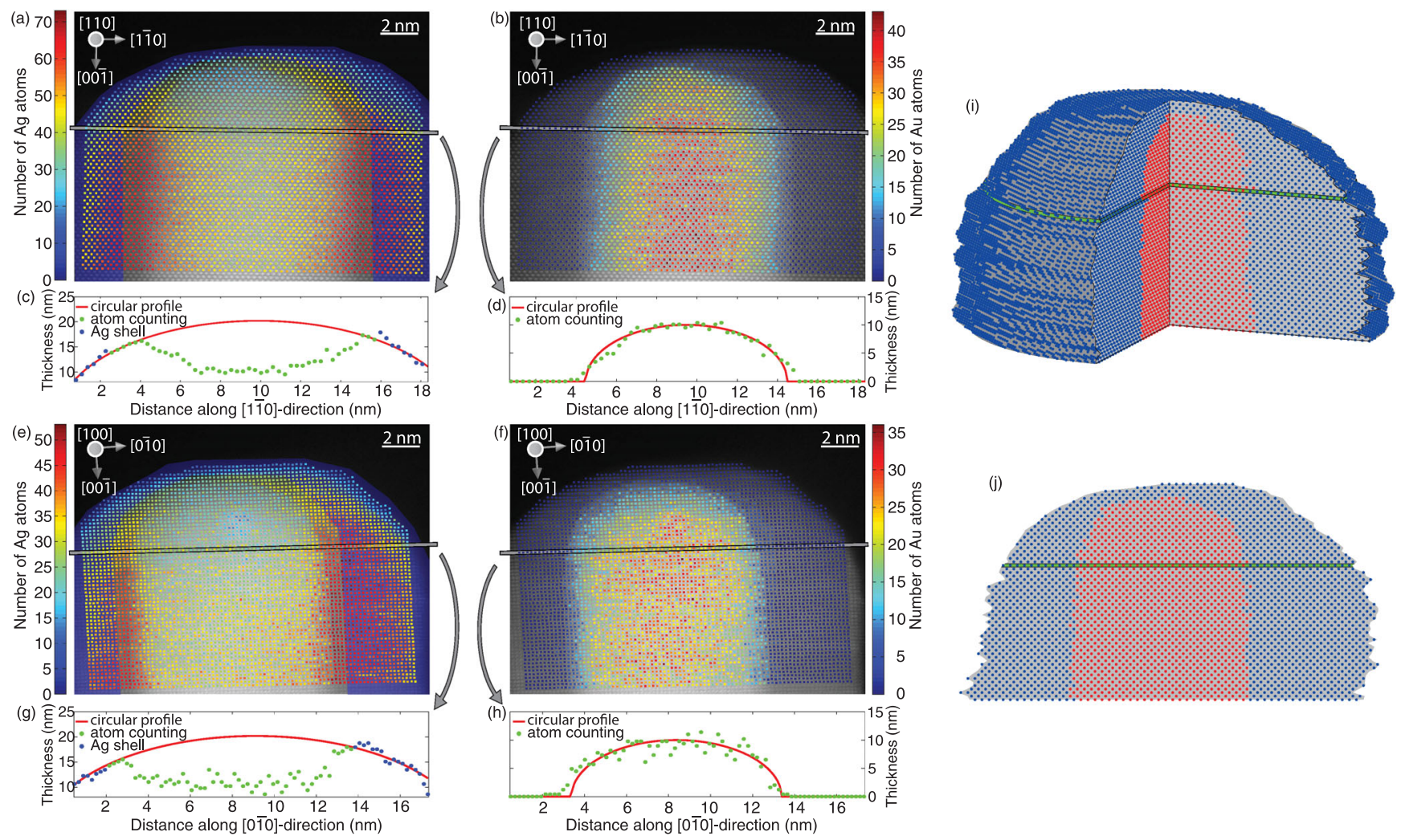

FIG. 3. Experimental HAADF STEM images of a Ag-coated Au nanorod. (a),(b) The number of (a) Ag and (b) Au atoms. (c),(d) The thickness profiles along the [110] direction of the single layer indicated in (a) and (b). (e),(f) The number of (e) Ag and (f) Au atoms from a second viewing direction. (g),(h) The thickness profiles along the [010] direction of the single layer indicated in (e) and (f). (c),(g) Circular profiles are fitted to the Ag shell, indicated in blue, to estimate the total thickness. (d) The cylindrical shape of the Au core is determined by fitting a second circular profile to atom-counting results along the [110] direction. (h) This fitted profile matches with the atom counting results along the $[0 \overline{1} 0]$ direction. (i) The reconstructed $3 \mathrm{D}$ model of the nanorod. (j) A cross section of the nanorod. The single layer is indicated in green (i),(j). 
that at the tips of the nanorod it is reasonable to assume that the shape of the nanorod is circular. Therefore, the thickness is estimated layer by layer by fitting a circular profile to counting results on the outermost pure Ag shell regions; see also Figs. 3(c) and 3(g). Here, counting results are presented on an absolute scale by combining statistical parameter estimation theory with frozen lattice image simulations of a pure Ag crystal [14,28]. For the thickness range suggested by the circular profile, more than $10^{22}$ different column configurations are possible, which can be limited to about 70000 by using the core-shell structure in which Au atoms should be located next to each other. This still requires an unrealistic number of image simulations, hampering atom counting in the absence of an accurate prediction model. The atomic lensing model now allows us to evaluate such nanostructures, as CPU computation time reduces by a factor of 1000. As shown in Fig. 1, the atomic lensing model starts to become less accurate beyond a thickness of about 25 atoms, and needs to be compensated. Two image simulations of isolated 55- and 75-atom-thick Ag columns containing 1 single Au atom indicated an error in the atomic lensing model of about $0.1 \%$ times the column thickness in number of atoms. This number has been used to compensate the predicted values. The atomic lensing model shows that the depth dependency is less pronounced in the thickness range of the nanorod. Therefore, the number of $\mathrm{Ag}$ and $\mathrm{Au}$ atoms can be computed from the direct comparison between the measured scattering cross sections and predicted values [see Figs. 3(a)-3(h)]. The Au counting results along the [1 10$]$ direction match to another circular profile, as shown in Fig. 3(d), confirming the expected cylindrical shape of the Au core for such nanorods. This circular profile also matches to the $\mathrm{Au}$ counting results along the [010] direction in Fig. 3(h), further validating the accuracy of the results. The 3D structure can be extracted from the fitted circular profiles in the two viewing directions. This suggests the presence of a Ag cylinder containing a cylindrical Au core located close to the center [see also Figs. 3(i) and 3(j)]. Therefore, this example shows that the atomic lensing model allows us to determine the 3D shape of heterogeneous nanocrystals from images taken from only two different viewing directions.

In the previous experimental example, the depth sensitivity of the scattering cross sections is too small compared to the presence of noise in the images, requiring the use of two viewing directions to retrieve the $3 \mathrm{D}$ reconstruction. However, for thin nanocrystals, where beam sensitivity may pose severe limitations in acquiring multiple images, the atomic lensing model opens up the possibility to extract 3D information from a single image. To illustrate this, a simulated image of a 15-atom-thick Au@Ag nanorod has been investigated. The nanorod contains a Au core, located close to the top, which is surrounded by a shell of Ag atoms, as shown in Fig. 4(a). Figure 4(b) shows the simulated image, where experimental conditions are mimicked by including source size broadening, a realistic electron dose, and the
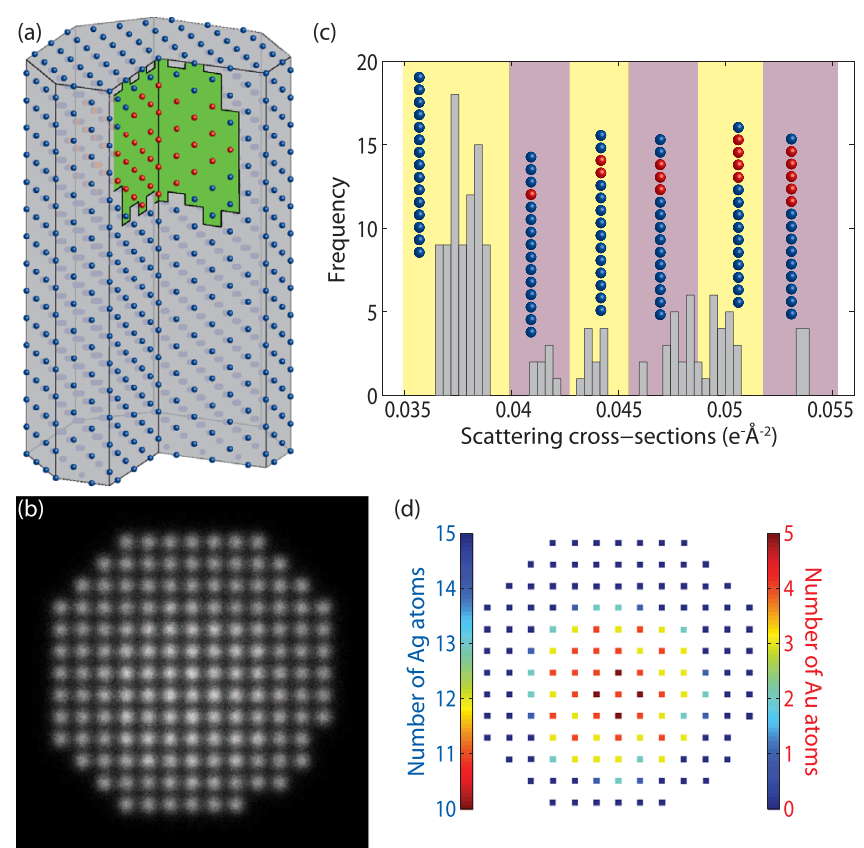

FIG. 4. (a) A 3D cross section of the Au@Ag nanorod used as input structure. Au and Ag atoms are represented by, respectively, red and blue spheres. The green area indicates the estimated range of possible depth locations of the gold atoms per atomic column. (b) An image simulation of the input structure viewed from the top, including Poisson noise and Gaussian source size broadening (with a FWHM of $0.7 \AA$ ). The incoming electron dose is $5 \times 10^{5}$ electrons $/ \AA^{2}$ (c) The histogram of the scattering cross sections of the different columns configurations. (d) The number of $\mathrm{Ag}$ and $\mathrm{Au}$ atoms per column. Image simulations are carried out by using the multislice approach with emission potential with parameters $U_{a}=300 \mathrm{kV}, C_{1}=-8.3 \mathrm{~nm}, C_{3}=0.035 \mathrm{~mm}$, $C_{5}=0 \mathrm{~mm}$, and $\alpha=22 \mathrm{mrad}$, and a detector collection range of 90-170 $\mathrm{mrad}[28]$.

presence of Poisson noise [42-44]. The histogram in Fig. 4(c) shows the scattering cross sections obtained by using statistical parameter estimation theory [12]. These values are compared to predicted scattering cross sections, using the atomic lensing model, to count the number of $\mathrm{Ag}$ and $\mathrm{Au}$ atoms per atomic column, as illustrated in Figs. 4(c) and 4(d). In this comparison, the number of possible column configurations has been limited by assuming a constant thickness of 15 atoms and a core-shell structure, in which Au atoms should be located next to each other. Next, the depth dependency of the scattering cross section is used to estimate the depth location of the Au atoms per atomic column. The uncertainty interval of depth locations, as indicated in green in Fig. 4(a), is controlled both by the precision on the measured scattering cross sections and the approximations in the atomic lensing model. This uncertainty is relatively higher in the case of low values for the scattering cross sections, explaining the larger uncertainty in the positioning of the columns containing only a few Au atoms. As illustrated in Fig. 4(a), the uncertainty is sufficiently low to conclude that the Au core is located in the 
top region of the nanorod. Therefore, this example demonstrates that by combining the atomic lensing model with prior knowledge about the thickness and shape, only one image is required to extract 3D information of thin-enough heterogeneous nanostructures.

In conclusion, a model for predicting scattering cross sections in HAADF STEM imaging is developed that is of great importance when quantitatively analyzing heterogeneous complex nanodevices. In this approach, only a limited number of images are required to retrieve 3D information when including prior knowledge about the structure under study. This method is successfully applied to experimental and simulated images of $\mathrm{Au} @ \mathrm{Ag}$ coreshell nanorods. Furthermore, the atomic lensing model is applicable to all sorts of 3D heterogeneous atomic column configurations. This model, therefore, pushes quantitative HAADF STEM imaging to the next level of unscrambling mixed elements, and can be used to help understand the properties of a wide range of nanomaterials.

The authors acknowledge financial support from the Research Foundation Flanders (FWO, Belgium) through Projects No. G.0374.13N, No. G.0368.15N, and No. G.0369.15N, and by grants to K. H. W. van den Bos and A. De Backer. S. Bals and N. Winckelmans acknowledge funding from the European Research Council (Starting Grant No. COLOURATOMS 335078). The research leading to these results has received funding from the European Union Seventh Framework Programme under Grant No. 312483-ESTEEM2. The authors are grateful to A. Rosenauer for providing the STEMsim program.

*Sandra.VanAert@uantwerpen.be

[1] P. M. Voyles, D. A. Muller, J. L. Grazul, P. H. Citrin, and H.-J. L. Gossmann, Nature (London) 416, 826 (2002).

[2] K. Tedsree, T. Li, S. Jones, C. W. A. Chan, K. M. K. Yu, P. A. J. Bagot, E. A. Marquis, G. D. Smith, and S. C. E. Tsang, Nat. Nanotechnol. 6, 302 (2011).

[3] R. Huang, Y.-H. Wen, Z.-Z. Zhu, and S.-G. Sun, J. Phys. Chem. C 116, 8664 (2012).

[4] S. Van Aert, K. J. Batenburg, M. D. Rossell, R. Erni, and G. Van Tendeloo, Nature (London) 470, 374 (2011).

[5] S. Bals, M. Casavola, M. A. van Huis, S. Van Aert, K. J. Batenburg, G. Van Tendeloo, and D. Vanmaekelbergh, Nano Lett. 11, 3420 (2011).

[6] S. Bals, S. Van Aert, C. P. Romero, K. Lauwaet, M. J. Van Bael, B. Schoeters, B. Partoens, E. Yücelen, P. Lievens, and G. Van Tendeloo, Nat. Commun. 3, 897 (2012).

[7] L. Jones, K. E. MacArthur, V. T. Fauske, A. T. J. van Helvoort, and P. D. Nellist, Nano Lett. 14, 6336 (2014).

[8] S. J. Pennycook and L. A. Boatner, Nature (London) 336, 565 (1988).

[9] P. D. Nellist and S. J. Pennycook, Ultramicroscopy 78, 111 (1999).

[10] P. Batson, N. Dellby, and O. Krivanek, Nature (London) 418, 617 (2002).
[11] R. Erni, M. D. Rossell, C. Kisielowski, and U. Dahmen, Phys. Rev. Lett. 102, 096101 (2009).

[12] S. Van Aert, J. Verbeeck, R. Erni, S. Bals, M. Luysberg, D. Van Dyck, and G. Van Tendeloo, Ultramicroscopy 109, 1236 (2009).

[13] H. E, K. E. MacArthur, T. J. Pennycook, E. Okunishi, A. J. D'Alfonso, N. R. Lugg, L. J. Allen, and P. D. Nellist, Ultramicroscopy 133, 109 (2013).

[14] S. Van Aert, A. De Backer, G. T. Martinez, B. Goris, S. Bals, G. Van Tendeloo, and A. Rosenauer, Phys. Rev. B 87, 064107 (2013).

[15] A. De Backer, G. T. Martinez, A. Rosenauer, and S. Van Aert, Ultramicroscopy 134, 23 (2013).

[16] G. T. Martinez, A. Rosenauer, A. De Backer, J. Verbeeck, and S. Van Aert, Ultramicroscopy 137, 12 (2014).

[17] H. Akamine, K. H. W. van den Bos, N. Gauquelin, S. Farjami, S. Van Aert, D. Schryvers, and M. Nishida, J. Alloys Compd. 644, 570 (2015).

[18] A. De Backer, G. T. Martinez, K. E. MacArthur, L. Jones, A. Béché, P. D. Nellist, and S. Van Aert, Ultramicroscopy 151, 56 (2015).

[19] G. T. Martinez, L. Jones, A. De Backer, A. Béché, J. Verbeeck, S. Van Aert, and P. D. Nellist, Ultramicroscopy 159, 46 (2015).

[20] G. T. Martinez, A. De Backer, A. Rosenauer, J. Verbeeck, and S. Van Aert, Micron 63, 57 (2014).

[21] T. Mehrtens, M. Schowalter, D. Tytko, P. Choi, D. Raabe, L. Hoffmann, H. Jönen, U. Rossow, A. Hangleiter, and A. Rosenauer, Appl. Phys. Lett. 102, 132112 (2013).

[22] L. D. Menard, S.-P. Gao, H. Xu, R. D. Twesten, A. S. Harper, Y. Song, G. Wang, A. D. Douglas, J. C. Yang, A. I. Frenkel et al., J. Phys. Chem. B 110, 12874 (2006).

[23] J. M. LeBeau and S. Stemmer, Ultramicroscopy 108, 1653 (2008).

[24] A. Rosenauer, K. Gries, K. Müller, A. Pretorius, M. Schowalter, A. Avramescu, K. Engl, and S. Lutgen, Ultramicroscopy 109, 1171 (2009).

[25] S. D. Findlay and J. M. LeBeau, Ultramicroscopy 124, 52 (2013).

[26] R. Ishikawa, A. R. Lupini, S. D. Findlay, T. Taniguchi, and S. J. Pennycook, Nano Lett. 14, 1903 (2014).

[27] J. Y. Zhang, J. Hwang, B. J. Isaac, and S. Stemmer, Sci. Rep. 5, 12419 (2015).

[28] A. Rosenauer and M. Schowalter, in Microscopy of Semiconducting Materials 2007 (Springer, New York, 2008), pp. 170-172.

[29] D. Van Dyck and M. Op de Beeck, Ultramicroscopy 64, 99 (1996).

[30] D. Van Dyck and J. H. Chen, Solid State Commun. 109, 501 (1999).

[31] P. Geuens and D. Van Dyck, Ultramicroscopy 93, 179 (2002).

[32] C. Rossouw, L. Allen, S. Findlay, and M. Oxley, Ultramicroscopy 96, 299 (2003).

[33] See Supplemental Material at http://link.aps.org/ supplemental/10.1103/PhysRevLett.116.246101 for a mathematical description of the atomic lensing model.

[34] P. Hartel, H. Rose, and C. Dinges, Ultramicroscopy 63, 93 (1996).

[35] A. Henglein, J. Phys. Chem. B 104, 2201 (2000). 
[36] M. Liu and P. Guyot-Sionnest, J. Phys. Chem. B 108, 5882 (2004).

[37] J. Chen, B. Wiley, Z.-Y. Li, D. Campbell, F. Saeki, H. Cang, L. Au, J. Lee, X. Li, and Y. Xia, Adv. Mater. 17, 2255 (2005).

[38] J. Becker, I. Zins, A. Jakab, Y. Khalavka, O. Schubert, and C. Sönnichsen, Nano Lett. 8, 1719 (2008).

[39] S. Gómez-Graña, J. Pérez-Juste, R. A. Alvarez-Puebla, A. Guerrero-Martínez, and L. M. Liz-Marzán, Adv. Opt. Mater. 1, 477 (2013).

[40] B. Goris, A. De Backer, S. Van Aert, S. Gómez-Graña, L. M. Liz-Marzán, G. Van Tendeloo, and S. Bals, Nano Lett. 13, 4236 (2013).
[41] S. Gómez-Graña, B. Goris, T. Altantzis, C. Fernández-López, E. Carbó-Argibay, A. Guerrero-Martínez, N. Almora-Barrios, N. López, I. Pastoriza-Santos, J. Pérez-Juste, S. Bals, G. Van Tendeloo, and L. M. Liz-Marzán, J. Phys. Chem. Lett. 4, 2209 (2013).

[42] P. D. Nellist and J. M. Rodenburg, Ultramicroscopy 54, 61 (1994).

[43] S. Van Aert, A. J. Den Dekker, D. Van Dyck, and A. van den Bos, Ultramicroscopy 90, 273 (2002).

[44] D. O. Klenov, S. D. Findlay, L. J. Allen, and S. Stemmer, Phys. Rev. B 76, 014111 (2007). 